\title{
THE HEAT KERNEL ON H-TYPE GROUPS
}

\author{
QIAOHUA YANG AND FULIU ZHU
}

(Communicated by David S. Tartakoff)

\begin{abstract}
In this paper we present an explicit calculation of the heat kernel for the sub-Laplacian on an H-type group $G$ by using irreducible unitary representations of $G$ and the heat kernel for the associated Hermite operator.
\end{abstract}

\section{INTRODUCTION}

As is well known, the heat kernel plays an important role in many problems in harmonic analysis. An explicit expression for the heat kernel on the Heisenberg group $H^{n}=\mathbb{C} \times \mathbb{R}$ was obtained by Hulanicki [9], Gaveau [7] and Staubach [11]. Gaveau [7] also obtained the heat kernel for free nilpotent Lie groups of step two. Cygan [6] obtained the heat kernel for all nilpotent Lie groups of step two. Zhu [13] used the method of the stochastic integral due to Gaveau to construct the heat kernel for the quaternionic Heisenberg groups. But neither Gaveau's expression for free nilpotent Lie groups nor Cygan's expression for arbitrary nilpotent Lie groups of step two were as explicit as those in the cases of Heisenberg groups and quaternionic Heisenberg groups.

The Hulanicki-Gaveau formula for the heat kernel on the Heisenberg groups has many interesting applications (see [13]). Although very impressive, these applications depend heavily on explicit expressions for the heat kernel. A natural and interesting question is : Are there other nilpotent Lie groups for which the expressions for the heat kernel are as explicit as those in the cases of the Heisenberg groups and quaternionic Heisenberg groups?

The aim of this paper is to look for such a formula for the heat kernel on H-type groups, a remarkable class of stratified groups of step two introduced by Kaplan [10]. On these groups there is a natural sub-Laplacian with an associated heat kernel. We use the method of Thangavelu [12], the irreducible unitary representation and the same argument as in [11], to calculate the heat kernel on the H-type groups and obtain a closed form of expression which closely resembles those of the heat kernel on Heisenberg groups and quaternionic Heisenberg groups. As we know, the H-type groups are the only nilpotent Lie groups on which an explicit formula for the heat kernel has been obtained up to now.

Received by the editors March 30, 2006.

2000 Mathematics Subject Classification. Primary 22E25; 35A08.

Key words and phrases. H-type groups, sub-Laplacian, heat kernel, Hermite operator.

The first author was supported by the National Science Foundation of China under grant number 10571044 .

(C) 2007 American Mathematical Society Reverts to public domain 28 years from publication 


\section{IRREDUCIBLE UNITARY REPRESENTATIONS}

We begin by describing the Lie groups and Lie algebras under consideration. An H-type group $G$ is a Carnot group of step two with the following properties: the Lie algebra $\mathfrak{g}$ of $G$ is endowed with an inner product $\langle$,$\rangle such that, if \mathfrak{z}$ is the center of $\mathfrak{g}$, then $\left[\mathfrak{z}^{\perp}, \mathfrak{z}^{\perp}\right]=\mathfrak{z}$ and, moreover, for every fixed $z \in \mathfrak{z}$, the map $J_{z}: \mathfrak{z}^{\perp} \rightarrow \mathfrak{z}^{\perp}$ defined by

$$
\left\langle J_{z}(v), \omega\right\rangle=\langle z,[v, \omega]\rangle, \quad \forall \omega \in \mathfrak{z}^{\perp}
$$

is an orthogonal map whenever $\langle z, z\rangle=1$ (see [3], [10]).

For reasons that will soon be clear we put $2 n=\operatorname{dim} \mathfrak{z}^{\perp}$ and $m=\operatorname{dim} \mathfrak{z}$. Fix $\lambda \in \mathfrak{z}^{*}$, the dual of $\mathfrak{z}$, and define the skew-symmetric linear mapping $B(\lambda)$ on $\mathfrak{z}^{\perp}$ by

$$
\langle B(\lambda) X, Y\rangle=\lambda([X, Y]) \quad \forall X, Y \in \mathfrak{z} .
$$

We denote by $z_{\lambda}$ the element of $\lambda \in \mathfrak{z}^{*}$ determined by

$$
\langle B(\lambda) X, Y\rangle=\lambda([X, Y])=\left\langle J_{z_{\lambda}}(X), Y\right\rangle .
$$

Denote the kernel of $B(\lambda)$ by $\mathfrak{r}_{\lambda}$, and let $\mathfrak{m}_{\lambda}$ be the orthogonal complement of $\mathfrak{r}_{\lambda}$ in $\mathfrak{z}^{\perp}$. Since $B(\lambda)$ is skew-symmetric, $\mathfrak{m}_{\lambda}$ is $B(\lambda)$-invariant and its dimension $\operatorname{dim} \mathfrak{m}_{\lambda}$ is even. Denote by $\Lambda$ the Zariski-open subset of $\mathfrak{z}^{*}$ of the vectors $\lambda$ for which $\operatorname{dim} \mathfrak{m}_{\lambda}$ is a maximum. From (2.1) we know that $\operatorname{dim} \mathfrak{m}_{\lambda}=\operatorname{dim} \mathfrak{z}^{\perp}=2 n$ and $\mathfrak{r}_{\lambda}=\{0\}$ for all $\lambda \in \Lambda$ and $\Lambda=\mathfrak{z}^{\perp} \backslash\{0\}$. For $\lambda, \mu \in \Lambda$, we put $\langle\lambda, \mu\rangle=\left\langle z_{\lambda}, z_{\mu}\right\rangle$ and $|\lambda|=\sqrt{\langle\lambda, \lambda\rangle}$. We denote by $\operatorname{Sym}_{m} B(\lambda)$ the symmetric function of degree $2 n$ in the roots of $B(\lambda)$.

Fix $\lambda$ in $\Lambda$. There are orthogonal vectors $E_{1}(\lambda), \cdots, E_{n}(\lambda), \bar{E}_{1}(\lambda), \cdots, \bar{E}_{n}(\lambda)$ in $\mathfrak{m}_{\lambda}$ such that

$$
B(\lambda) E_{i}(\lambda)=|\lambda| \bar{E}_{i}(\lambda) \quad \text { and } \quad B(\lambda) \bar{E}_{i}(\lambda)=-|\lambda| E_{i}(\lambda),
$$

and $\operatorname{Sym}_{2 n} B(\lambda)=|\lambda|^{2 n}$. We may further decompose $\mathfrak{z}^{\perp}$. Denote by $\mathfrak{x}_{\lambda}$ and $\mathfrak{y}_{\lambda}$ the subspaces $\operatorname{span}\left\{E_{1}(\lambda), \cdots, E_{n}(\lambda)\right\}$ and $\operatorname{span}\left\{\bar{E}_{1}(\lambda), \cdots, \bar{E}_{n}(\lambda)\right\}$, respectively. Then we may write $V$ in $\mathfrak{z}^{\perp}$ as $X+Y$ with $X \in \mathfrak{x}_{\lambda}$ and $Y \in \mathfrak{y}_{\lambda}$. With respect to this basis, the group law on an H-type group $G$ has the form

$$
\begin{aligned}
(z, t) \circ\left(z^{\prime}, t^{\prime}\right) & =(x, y, t) \circ\left(x^{\prime}, y^{\prime}, t^{\prime}\right) \\
& =\left(\begin{array}{c}
z_{i}+z_{i}^{\prime}, \quad i=1,2, \cdots, 2 n \\
t_{j}+t_{j}+\frac{1}{2}\left\langle z, U^{(j)} z^{\prime}\right\rangle, \quad j=1,2, \cdots, m
\end{array}\right)
\end{aligned}
$$

where $z=(x, y) \in R^{2 n}$ and the matrices $U^{(1)}, U^{(2)}, \cdots, U^{(2 n)}$ satisfy the following conditions:

(1) $U^{j}$ is an $m \times m$ skew-symmetric and orthogonal matrix, for every $j=$ $1,2, \cdots, 2 n$;

(2) $U^{(i)} U^{(j)}+U^{(j)} U^{(i)}=0$ for every $i, j \in\{1,2, \cdots, 2 n\}$ with $i \neq j$.

The irreducible unitary representations parameterized by $\lambda \in \Lambda$ may be described as follows (see [1], [5]):

$$
\left(\pi_{\lambda}(x, y, t) \phi\right)(\xi)=e^{i \sum_{j=1}^{m} \lambda_{j} t_{j}+i \sum_{j=1}^{n}\left(x_{j} \xi_{j}+\frac{1}{2} x_{j} y_{j}\right)|\lambda|} \phi(\xi+y),
$$

for all $\phi \in L^{2}\left(\mathfrak{y}_{\lambda}\right)$. For $f \in L^{1}(G)$ and $\lambda \in \Lambda$, its Fourier transform $\widehat{f}(\lambda)$ is the operator-valued function defined by

$$
\widehat{f}(\lambda)=\int_{\mathfrak{z}} \int_{\mathfrak{y}_{\lambda}} \int_{\mathfrak{x}_{\lambda}} f(x, y, t) \pi_{\lambda}(x, y, t) d x d y d t .
$$


This means that for each $\varphi, \psi \in L^{2}\left(\mathfrak{y}_{\lambda}\right)$,

$$
(\widehat{f}(\lambda) \varphi, \psi)=\int_{\mathfrak{z}} \int_{\mathfrak{y}_{\lambda}} \int_{\mathfrak{x}_{\lambda}} f(x, y, t)\left(\pi_{\lambda}(x, y, t) \varphi, \psi\right) d x d y d t .
$$

We now describe the sub-Laplacian on an H-type group $G$ (see also [3]). The vector fields in the algebra $\mathfrak{g}$ of $G=\left(\mathbf{R}^{2 n+m}, \circ\right)$ that agrees at the origin with $\frac{\partial}{\partial x_{j}}, \frac{\partial}{\partial y_{j}}(j=1,2, \cdots, n)$ are given by

$$
\begin{gathered}
X_{j}=\frac{\partial}{\partial x_{j}}+\frac{1}{2} \sum_{k=1}^{m}\left(\sum_{l=1}^{2 n} z_{l} U_{l, j}^{(k)}\right) \frac{\partial}{\partial t_{k}}, \\
Y_{j}=\frac{\partial}{\partial y_{j}}+\frac{1}{2} \sum_{k=1}^{m}\left(\sum_{l=1}^{2 n} z_{l} U_{l, j+n}^{(k)}\right) \frac{\partial}{\partial t_{k}}
\end{gathered}
$$

with $\left(U_{l, j}^{(k)}\right)_{2 n \times 2 n}=U^{(k)}$. The Kohn's sub-Laplacian on the H-type group $G$ is given by

$$
\mathcal{L}=-\sum_{j=1}^{n}\left(X_{j}^{2}+Y_{j}^{2}\right)=-\Delta_{z}-\frac{1}{4}|z|^{2} \Delta_{t}-\sum_{k=1}^{m}\left\langle z, U^{(k)} \nabla_{z}\right\rangle \frac{\partial}{\partial t_{k}},
$$

where

$$
\Delta_{z}=\sum_{j=1}^{2 n} \frac{\partial^{2}}{\partial z_{j}^{2}}=\sum_{j=1}^{n}\left(\frac{\partial^{2}}{\partial x_{j}^{2}}+\frac{\partial^{2}}{\partial y_{j}^{2}}\right), \quad \Delta_{t}=\sum_{k=1}^{m} \frac{\partial^{2}}{\partial t_{j}^{2}}
$$

and

$$
\nabla_{z}=\left(\nabla_{x}, \nabla_{y}\right)^{T}=\left(\frac{\partial}{\partial x_{1}},, \cdots, \frac{\partial}{\partial x_{n}}, \frac{\partial}{\partial y_{1}}, \cdots, \frac{\partial}{\partial y_{n}}\right)^{T}
$$

We recall that this essentially selfadjoint positive operator does not depend on the choice of an orthonormal basis of $\mathfrak{z}^{\perp}$. Moreover, on functions $\widetilde{u}(z, t)=u(|z|, t)$, we obtain (see [3])

$$
\left\langle z, U^{(k)} \nabla_{z}\right\rangle \widetilde{u}(z, t)=0, \quad k=1,2, \cdots, m,
$$

and hence $\mathcal{L}$ has the form

$$
\mathcal{L} \widetilde{u}(z, t)=-\Delta_{z} \widetilde{u}(z, t)-\frac{1}{4}|z|^{2} \Delta_{u} \widetilde{u}(z, t) .
$$

The representation $\pi_{\lambda}$ of $G$ determines a representation $\pi_{\lambda}^{*}$ of its Lie algebra $\mathfrak{g}$ on the space of $C^{\infty}$ vectors. Recall that $f$ is said to be a $C^{\infty}$ vector for the representation $\pi_{\lambda}$ if $(x, y, t) \mapsto \pi_{\lambda} f$ is a $C^{\infty}$ function from $G$ into the Hilbert space. The representation $\pi_{\lambda}^{*}$ is defined by

$$
\pi_{\lambda}^{*}(X) f=\left(\frac{d}{d t} \pi_{\lambda}(\exp t X) f\right)_{t=0}
$$

for every $X$ in the Lie algebra $\mathfrak{g}$. We can then extend $\pi_{\lambda}^{*}$ to the universal enveloping algebra of left-invariant differential operators on $G$. If $A$ is any such operator, then a simple computation shows that

$$
A\left(\pi_{\lambda}(x, y, t) f, g\right)=\left(\pi_{\lambda}(x, y, t) \pi_{\lambda}^{*}(A) f, g\right) .
$$

Therefore, one way of obtaining entry functions that are eigenfunctions of $A$ is to take $f$ to be an eigenfunction of the operator $\pi_{\lambda}^{*}(A)$. An easy calculation yields that

$$
\pi_{\lambda}^{*}\left(X_{j}\right)=i|\lambda| \xi_{j}, \quad \pi_{\lambda}^{*}\left(Y_{j}\right)=\frac{\partial}{\partial \xi_{j}}
$$


for $j=1,2, \cdots, n$, so that

$$
H(\lambda)=\pi_{\lambda}^{*}(\mathcal{L})=-\sum_{j=1}^{n} \frac{\partial^{2}}{\partial \xi_{j}^{2}}+|\lambda|^{2}|\xi|^{2}
$$

is the Hermite operator. The eigenfunctions of $H(\lambda)$ are given by

$$
\Phi_{\alpha}^{\lambda}(\xi)=|\lambda|^{\frac{n}{4}} \Phi_{\alpha}(\sqrt{|\lambda|} \xi), \quad \alpha=\left(\alpha_{1}, \cdots, \alpha_{n}\right),
$$

where $\Phi_{\alpha}(\xi)$ is the product $\psi_{\alpha_{1}}\left(\xi_{1}\right) \cdots \psi_{\alpha_{n}}\left(\xi_{n}\right)$ and $\psi_{\alpha_{j}}\left(\xi_{j}\right)(j=1, \cdots, n)$ is the eigenfunction of $-\frac{\partial^{2}}{\partial \xi_{j}^{2}}+\xi_{j}^{2}$ with eigenvalue $2 \alpha_{i}+1$ (see [4], [12]). Note that

$$
H(\lambda) \Phi_{\alpha}^{\lambda}=(2|\alpha|+n)|\lambda| \Phi_{\alpha}^{\lambda}, \quad|\alpha|=\alpha_{1}+\cdots+\alpha_{n} ;
$$

therefore,

$$
\mathcal{L}\left(\pi_{\lambda}(x, y, t) \Phi_{\alpha}^{\lambda}, \Phi_{\beta}^{\lambda}\right)=(2|\alpha|+n)|\lambda|\left(\pi_{\lambda}(x, y, t) \Phi_{\alpha}^{\lambda}, \Phi_{\beta}^{\lambda}\right) .
$$

Thus the entry functions $\left(\pi_{\lambda}(x, y, t) \Phi_{\alpha}^{\lambda}, \Phi_{\beta}^{\lambda}\right)$ as $\alpha, \beta$ range over $\mathbf{N}^{n}$ give a family of eigenfunctions for the sub-Laplacian.

Let $\mathcal{L}_{\lambda}$ be the operator defined by the relation

$$
\mathcal{L}\left(e^{i \sum_{j=1}^{m} \lambda_{j} t_{j}} f(x, y)\right)=e^{i \sum_{j=1}^{m} \lambda_{j} t_{j}} \mathcal{L}_{\lambda} f(x, y) .
$$

Explicitly, $\mathcal{L}_{\lambda}$ is given by the expression

$$
\mathcal{L}_{\lambda}=-\Delta_{z}+\frac{1}{4}|\lambda|^{2}|z|^{2}-\mathcal{N},
$$

where $\mathcal{N}=i \sum_{k=1}^{m} \lambda_{k}\left\langle z, U^{(k)} \nabla_{z}\right\rangle$. Put $\pi_{\lambda}(x, y)=\pi_{\lambda}(x, y, 0)$. Since

$$
\left(\pi_{\lambda}(x, y, t) \Phi_{\alpha}^{\lambda}, \Phi_{\beta}^{\lambda}\right)=e^{i \sum_{j=1}^{m} \lambda_{j} t_{j}}\left(\pi_{\lambda}(x, y) \Phi_{\alpha}^{\lambda}, \Phi_{\beta}^{\lambda}\right),
$$

the functions

$$
\Phi_{\alpha \beta}^{\lambda}(z)=(2 \pi)^{-\frac{n}{2}}|\lambda|^{\frac{n}{2}}\left(\pi_{\lambda}(x, y) \Phi_{\alpha}^{\lambda}, \Phi_{\beta}^{\lambda}\right)
$$

are eigenfunctions of the operator $\mathcal{L}_{\lambda}$ :

$$
\mathcal{L}_{\lambda} \Phi_{\alpha \beta}^{\lambda}(z)=(2|\alpha|+n)|\lambda| \Phi_{\alpha \beta}^{\lambda}(z) .
$$

They are also eigenfunctions of the operators $-\Delta_{z}+\frac{1}{4}|\lambda|^{2}|z|^{2}$ (see [12], page 54):

$$
\left(-\Delta_{z}+\frac{1}{4}|\lambda|^{2}|z|^{2}\right) \Phi_{\alpha \beta}^{\lambda}(z)=(|\alpha|+|\beta|+n)|\lambda| \Phi_{\alpha \beta}^{\lambda}(z) .
$$

From (2.4) and (2.5) we obtain

$$
\mathcal{N} \Phi_{\alpha \beta}^{\lambda}(z)=(|\alpha|-|\beta|)|\lambda| \Phi_{\alpha \beta}^{\lambda}(z) .
$$

We now define the Laguerre functions $\varphi_{k}^{n-1}(z)$ on $\mathbb{R}^{2 n}$ by

$$
\varphi_{k}^{n-1}(z)=L_{k}^{n-1}\left(\frac{1}{2}|z|^{2}\right) e^{-\frac{1}{4}|z|^{2}}
$$

where

$$
L_{k}^{n-1}(s)=e^{s} t^{-s} \frac{1}{k !} \frac{d^{n-1}}{d s^{n-1}}\left(e^{-s} s^{n-1+k}\right) \quad \text { for } s>0 .
$$

We also define $\varphi_{k, \lambda}^{n-1}(z)=\varphi_{k}^{n-1}(\sqrt{\lambda} z)$ for $|\lambda| \neq 0$. Then $\varphi_{k, \lambda}^{n-1}$ satisfies (see [12], page 58)

$$
\varphi_{k, \lambda}^{n-1}(z)=(2 \pi)^{\frac{n}{2}}|\lambda|^{\frac{n}{2}} \sum_{|\alpha|=k} \Phi_{\alpha \alpha}^{\lambda}(z) .
$$




\section{THE HEAT KERNEL}

In this section, we calculate the heat kernel of the sub-Laplacian $\mathcal{L}$, given by

$$
\begin{aligned}
\mathcal{L} & =-\sum_{j=1}^{n}\left(X_{j}^{2}+Y_{j}^{2}\right)=-\Delta_{z}-\frac{1}{4}|z|^{2} \Delta_{t}-\sum_{k=1}^{m}\left\langle z, U^{(k)} \nabla_{z}\right\rangle \frac{\partial}{\partial t_{k}} \\
& =-\sum_{j=1}^{n}\left(\frac{\partial^{2}}{\partial x_{j}^{2}}+\frac{\partial^{2}}{\partial y_{j}^{2}}\right)-\frac{1}{4}\left(|x|^{2}+|y|^{2}\right) \sum_{k=1}^{m} \frac{\partial^{2}}{\partial t_{j}^{2}}-\sum_{k=1}^{m}\left\langle z, U^{(k)} \nabla_{z}\right\rangle \frac{\partial}{\partial t_{k}} .
\end{aligned}
$$

We observe that $\left\{X_{j}, Y_{j}\right\}_{j=1}^{n}$ and their first-order commutators span the whole Lie algebra $\mathfrak{g}$. Thus the sub-Laplacian is an example of a step-two hypoelliptic operator, studied in Hörmander [8].

We start by looking at the heat equation:

$$
\begin{aligned}
& \frac{\partial p_{s}(x, y, t)}{\partial s}=-\mathcal{L} p_{s}(x, y, t), \quad s>0, x, y \in \mathbb{R}^{n}, t \in \mathbb{R}^{m}, \\
& p_{0}(x, y, t)=\delta_{0}
\end{aligned}
$$

where $\delta_{0}$ is the Dirac mass at the origin. The calculation of the heat kernel proceeds along the same lines as [11]. Taking the Fourier transform in the $t$ variable yields

$$
\begin{aligned}
& \frac{\partial p_{s}^{\lambda}}{\partial s}=-\sum_{j=1}^{n}\left(\frac{\partial^{2}}{\partial x_{j}^{2}}+\frac{\partial^{2}}{\partial y_{j}^{2}}\right) p_{s}^{\lambda}+\frac{1}{4}\left(|x|^{2}+|y|^{2}\right)|\lambda|^{2} p_{s}^{\lambda}-i \sum_{k=1}^{m} \lambda_{k}\left\langle z, U^{(k)} \nabla_{z}\right\rangle p_{s}^{\lambda}, \\
& p_{0}^{\lambda}(x, y, \lambda)=\delta \otimes 1_{\lambda},
\end{aligned}
$$

where

$$
p_{s}^{\lambda}=\int_{\mathbf{R}^{m}} e^{i \lambda \cdot t} p_{s}(x, y, t) d t
$$

and $\delta=\delta(x, y)$ is the Dirac mass in $\mathbb{R}^{2 n}$.

We rewrite the equation as

$$
\frac{\partial p_{s}^{\lambda}}{\partial s}=\left(-\Delta_{z}+\frac{1}{4}|\lambda|^{2}|z|^{2}\right) p_{s}^{\lambda}-\mathcal{N} p_{s}^{\lambda}
$$

where $\mathcal{N}=i \sum_{k=1}^{m} \lambda_{k}\left\langle z, U^{(k)} \nabla_{z}\right\rangle$, introduced earlier.

First we shall show that the Lie bracket $\left[-\Delta_{z}+\frac{1}{4}|\lambda|^{2}|z|^{2}, \mathcal{N}\right]$ is zero.

Lemma 3.1. $\left[-\Delta_{z}+\frac{1}{4}|\lambda|^{2}|z|^{2}, \mathcal{N}\right]=0$.

Proof. Since $\mathcal{N}=i \sum_{k=1}^{m} \lambda_{k}\left\langle z, U^{(k)} \nabla_{z}\right\rangle$, a simple computation shows that

$$
\frac{\partial}{\partial z_{j}} \mathcal{N}=i \sum_{k=1}^{m} \lambda_{k}\left(\sum_{l=1}^{2 n} U_{j, l}^{(k)} \frac{\partial}{\partial z_{l}}\right)+\mathcal{N} \frac{\partial}{\partial z_{j}}
$$

and

$$
\frac{\partial^{2}}{\partial z_{j}^{2}} \mathcal{N}=2 i \sum_{k=1}^{m} \lambda_{k}\left(\sum_{l=1}^{2 n} U_{j, l}^{(k)} \frac{\partial}{\partial z_{l}}\right) \frac{\partial}{\partial z_{j}}+\mathcal{N} \frac{\partial^{2}}{\partial z_{j}^{2}} .
$$

Therefore we obtain

$$
\Delta_{z} \mathcal{N}=\sum_{j=1}^{2 n} \frac{\partial^{2}}{\partial z_{j}^{2}} \mathcal{N}=2 i \sum_{k=1}^{m} \lambda_{k}\left(\sum_{j=1}^{2 n} \sum_{l=1}^{2 n} U_{j, l}^{(k)} \frac{\partial^{2}}{\partial z_{l} z_{j}}\right)+\mathcal{N} \Delta_{z}
$$


Recalling $U^{k}(k=1,2, \cdots, m)$ is skew-symmetric,

$$
\sum_{j=1}^{2 n} \sum_{l=1}^{2 n} U_{j, l}^{(k)} \frac{\partial^{2}}{\partial z_{l} z_{j}}=0, \quad k=1,2, \cdots, m,
$$

we have, from (3.1),

$$
\Delta_{z} \mathcal{N}=\mathcal{N} \Delta_{z}
$$

Thus

$$
\begin{aligned}
{\left[-\Delta_{z}+\frac{1}{4}|\lambda|^{2}|z|^{2}, \mathcal{N}\right] } & =-\Delta_{z} \mathcal{N}+\frac{1}{4}|\lambda|^{2}|z|^{2} \mathcal{N}+\mathcal{N} \Delta_{z}-\mathcal{N} \cdot \frac{1}{4}|\lambda|^{2}|z|^{2} \\
& =\frac{1}{4}|\lambda|^{2}|z|^{2} \mathcal{N}-\frac{1}{4}|\lambda|^{2} \mathcal{N}\left(|z|^{2}\right)-\frac{1}{4}|\lambda|^{2}|z|^{2} \mathcal{N} \\
& =0
\end{aligned}
$$

Here we use the fact $\mathcal{N}|z|^{2}=0($ see $(2.3))$.

Lemma 3.1 is quite useful and enables us to write

$$
e^{s\left(-\Delta_{z}+\frac{1}{4}|\lambda|^{2}|z|^{2}-\mathcal{N}\right)}=e^{-s \mathcal{N}} e^{s\left(-\Delta_{z}+\frac{1}{4}|\lambda|^{2}|z|^{2}\right)} .
$$

So we are led to study the heat semigroup $e^{s\left(-\Delta_{z}+\frac{1}{4}|\lambda|^{2}|z|^{2}\right)}$.

Let $h_{s}(x, y)$ be the heat kernel of the Hermite operator $-\Delta_{z}+\frac{1}{4}|\lambda|^{2}|z|^{2}$, that is,

$$
\frac{\partial h_{s}(x, y)}{\partial s}=\left(-\Delta_{z}+\frac{1}{4}|\lambda|^{2}|z|^{2}\right) h_{s}(x, y)
$$

with

$$
\lim _{s \rightarrow 0} \int_{\mathbf{R}^{m}} h_{s}(x, y) f(x, y)=f(0,0) .
$$

An explicit calculation of $h_{s}(x, y)$ is given by (see e.g. [4], Theorem 2.1)

$$
h_{s}(x, y)=(4 \pi)^{-n}\left(\frac{|\lambda|}{\sinh |\lambda| s}\right)^{n} \exp \left\{-\frac{|\lambda||z|^{2}}{4} \operatorname{coth}|\lambda| s\right\}
$$

and hence (see [12], page 85, 2.8.7)

$$
h_{s}(x, y)=(2 \pi)^{-n}|\lambda|^{n} \sum_{k=0}^{\infty} e^{-(2 k+n)|\lambda| s} \varphi_{k, \lambda}^{n-1},
$$

where $\varphi_{k, \lambda}^{n-1}$ was introduced earlier.

From (2.6), (2.7) and (3.3), the explicit spectral decomposition of $\mathcal{N}$ is given by

$$
\begin{aligned}
\mathcal{N} h_{s}(x, y) & =(2 \pi)^{-n}|\lambda|^{n} \sum_{k=0}^{\infty} e^{-(2 k+n)|\lambda| s} \mathcal{N} \varphi_{k, \lambda}^{n-1} \\
& =(2 \pi)^{-n}|\lambda|^{n} \sum_{k=0}^{\infty} e^{-(2 k+n)|\lambda| s}(2 \pi)^{-\frac{n}{2}}|\lambda|^{\frac{n}{2}} \mathcal{N} \sum_{|\alpha|=k} \Phi_{\alpha \alpha}^{\lambda}(z) \\
& =(2 \pi)^{-n}|\lambda|^{n} \sum_{k=0}^{\infty} e^{-(2 k+n)|\lambda| s}(2 \pi)^{-\frac{n}{2}}|\lambda|^{\frac{n}{2}} \cdot 0 \\
& =0 .
\end{aligned}
$$


Therefore, from (3.2), (3.3) and (3.4), $p_{s}^{\lambda}$ is given by

$$
\begin{aligned}
p_{s}^{\lambda} & =e^{-s \mathcal{N}} e^{s\left(-\Delta_{z}+\frac{1}{4}|\lambda|^{2}|z|^{2}\right)}\left(\delta \otimes 1_{\lambda}\right) \\
& =e^{-s \mathcal{N}} h_{s}(x, y) \\
& =h_{s}(x, y) .
\end{aligned}
$$

This gives us, after taking the inverse Fourier transform, the heat kernel for the sub-Laplacian:

$$
p_{s}(x, y, t)=(2 \pi)^{-m}(4 \pi)^{-n} \int_{\mathbf{R}^{m}}\left(\frac{|\lambda|}{\sinh |\lambda| s}\right)^{n} \exp \left\{-\frac{|\lambda||z|^{2}}{4} \operatorname{coth}|\lambda| s-i \lambda \cdot t\right\} d \lambda .
$$

We summarize the computations and the formula as a theorem:

Theorem 3.2. The heat kernel of the sub-Laplacian on an H-type group is given by

$$
p_{s}(x, y, t)=(2 \pi)^{-m}(4 \pi)^{-n} \int_{\mathbf{R}^{m}}\left(\frac{|\lambda|}{\sinh |\lambda| s}\right)^{n} \exp \left\{-\frac{|\lambda||z|^{2}}{4} \operatorname{coth}|\lambda| s-i \lambda \cdot t\right\} d \lambda .
$$

\section{ACKNOWLEDGMENTS}

The authors thanks the referee for his/her careful reading and very useful comments which improved the final version of this paper.

\section{REFERENCES}

1. F. Astengo, M. Cowling, B. Di Blasio, M. Sundari, Hardy's uncertainty principle on certain Lie groups, J. London Math. Soc., 62 (2000), 461-472. MR1783638 (2002b:22018)

2. R. Beals, B. Gaveau, P. Greiner, Complex Hamiltonian mechanics and parametrices for subelliptic Laplacians, Bull. Sci. Math. 121 (1997), 1-36. MR1431098 (98b:35032a)

3. A. Bonfiglioli, F. Uguzzoni, Nonlinear Liouville theorems for some critical problems on H-type groups, J. Functional Analysis, 207 (2004), 161-215. MR2027639 (2004k:35057)

4. D.C. Chang, J.Z. Tie, A note on Hermite and subelliptic operators, Acta Math. Sin., 21 (2005), 803-818. MR2156956 (2006e:42013)

5. L.J. Corwin, F.P. Greenleaf, Representations of nilpotent Lie groups and their applications. 1: Basic theory and examples, Cambridge Studies in Advanced Mathematics 18, Cambridge University Press, 1990. MR1070979 (92b:22007)

6. J. Cygan, Heat kernels for class 2 nilpotent groups, Studia Math., 64 (1979), 227-238. MR544727 (82b:22016)

7. B. Gaveau, Principe de moindre action, propagation de la chaleur et estimées sous elliptiques sur certains groupes nilpotents, Acta Math., 139 (1977), 95-153. MR0461589 (57:1574)

8. L. Hörmander, Hypoelliptic second order differential equations, Acta Math., Uppsala, 119 (1967), 147-171. MR0222474 (36:5526)

9. A. Hulanicki, The distribution of energy of the Brownian motion in the Gaussian field and analytic hypoellipticity of certain subelliptic operators on the Heisenberg group, Studia Math., 56 (1976), 165-173. MR0418257 (54:6298)

10. A. Kaplan, Fundamental solutions for a class of hypoelliptic PDE generated by composition of quadratic forms, Trans. Amer. Math. Soc., 258 (1980), 147-153. MR554324 (81c:58059)

11. W. Staubach, Wiener path integrals and the fundamental solution for the Heisenberg Laplacian, J. d'Analyse Math., 91 (2003), 389-400. MR2037416 (2005m:35052)

12. S. Thangavelu, An introduction to the uncertainty principle: Hardy's theorem on Lie groups, Progress in Mathematics, 217, Birkhäuser, Boston, 2004. MR2008480 (2004j:43007)

13. Fuliu Zhu, The heat kernel and the Riesz transforms on the quaternionic Heisenberg groups, Pacific J. Math. 209 (2003), 175-199. MR1973940 (2004e:43013) 
School of Mathematics and Statistics, Wuhan University, Wuhan, 430072, People's Republic of China

Current address: Wuhan Institute of Physics and Mathematics, The Chinese Academy of Sciences, P.O. Box 71010, Wuhan 430071, People's Republic of China

E-mail address: qaohyang2465@yahoo.com.cn

School of Mathematics and Statistics, Wuhan University, Wuhan, 430072, People's Republic of ChinA

E-mail address: flzhu@whu.edu.cn 